\title{
Scalar curvature rigidity of certain symmetric spaces
}

\author{
Maung Min-Oo*
}

May 1995

\begin{abstract}
This is a short selected survey of results on scalar curvature rigidity of certain symmetric spaces, in particular, for the Euclidean, hyperbolic and spherical metrics. The proofs, all of which use spinors, are sketched, with an attempt to show the basic underlying structure.
\end{abstract}

\section{Local Rigidity}

Since the results in this section have their origins in the proof of the positive mass conjecture, we will begin by describing briefly the relevant gravitational background.

In general relativity, there is no satisfactory notion of total energy, since the energy of the gravitational field itself is described purely in terms of geometry and does not contribute directly to the local stress-energy-momentum tensor $T_{i j}$. However, in an asymptotically flat space time describing an isolated system like a star or a black hole, where the gravitational field approaches ordinary Newtonian gravity with respect to a background inertial coordinate system at infinity, one can define the total mass, or more relativistically, the total energymomentum four-vector of the system by asymptotic comparison with Newtonian theory at large distances.

More precisely, we define an asymptotically Euclidean space-like hypersurface to be a 3dimensional oriented Riemannian manifold $(M, g)$ isometrically imbedded in 4-dimensional space-time whose first and second fundamental forms $g_{i j}$ and $h_{i j}$ satisfy the following asymptotic conditions:

(A) There is a compact set $K \subset M$ so that $M-K$ is a finite disjoint union of ends, each diffeomorphic to the complement of a closed ball in $\mathbb{R}^{3}$ and using the standard coordinates given by this diffeomorphism, $g$ and $h$ have the asymptotic behaviour:

$$
\partial_{\alpha}\left(g_{i j}-\delta_{i j}\right) \in O\left(r^{-1-|\alpha|}\right) \text { for }|\alpha| \leq 2
$$

${ }^{*}$ This work was partially supported by an NSERC grant OGP-7873. 
and

$$
\partial_{\beta}\left(h_{i j}\right) \in O\left(r^{-2-|\beta|}\right) \text { for }|\beta| \leq 1
$$

These are not the optimal decay rates and we refer to [B] for refinements and also for a discussion of the independence of the ADM-mass, from the choice of the coordinate system at infinity. ADM stands for Arnowitt, Deser and Misner and their definition of the total energy-momentum $\left(E, P_{j}\right)$ of an asymptotically Euclidean space-like slice is:

$$
\begin{gathered}
E=\frac{1}{16 \pi G} \lim _{r \rightarrow \infty} \oint_{S(r)}\left(\partial_{k} g_{i k}-\partial_{i} g_{k k}\right) d \sigma^{i} \\
P_{j}=\frac{1}{8 \pi G} \lim _{r \rightarrow \infty} \oint_{S(r)}\left(h_{i j}-\delta_{i j} h_{k k}\right) d \sigma^{i}
\end{gathered}
$$

where $S(r)$ is the Euclidean sphere of radius $r$ and the integrals are defined for each end. For the important prototypical example of the Schwarzschild metric, this definition of course recovers the usual mass that appears in the metric and $P=0$.

The next important physical assumption assumption is the following dominant energy condition for the local mass density $T$ :

(B) For each time like vector $e_{0}$ transversal to $M, T\left(e_{0}, e_{0}\right) \geq 0$ and $T\left(e_{0}, \quad\right)$ ia a nonspace-like covector. This implies that for any adapted orthonormal frame $\left(e_{0}, e_{1}, e_{2}, e_{3}\right)$ with $e_{0}$ normal and $e_{1}, e_{2}, e_{3}$ tangential to $M$, we have the inequalities:

$$
T^{00} \geq\left|T^{\mu \nu}\right| \text { for all } 0 \leq \mu, \nu \leq 3
$$

and

$$
T^{00} \geq\left(-T_{0 k} T^{0 k}\right)^{1 / 2}
$$

We of course also assume that space time satisfies Einstein's field equations:

$$
R_{\mu \nu}-\frac{1}{2} R g_{\mu \nu}=8 \pi G T_{\mu \nu}
$$

Given these assumptions, the positive energy theorem states that

Theorem 1 An asymptotically Euclidean space-like hypersurface in a space-time satisfying Eistein's equation and the dominant energy condition has non-negative total energy in the sense that $E \geq|P|$ for each end. Moreover if $E=0$ for some end, then there is exactly one end and $M$ is isometric to flat Euclidean space.

After several attempts by relativists, who established several special cases, the first complete proof of this result was achieved by Schoen and Yau [SY 2,3,4], using minimal surface techniques. Subsequently, Witten [W] found a completely different proof using harmonic 
spinors. It should be noted that some analytical subtleties in Witten's paper were clarified later by Parker and Taubes [PT]. In this section, we will present some results which are proved by extending Witten's method to other asymptotic background geometries.

Parallel to this development in relativity and, of course closely related to it, was the famous problem about the existence and rigidity of positive scalar curvature metrics on the torus. Here again there were two breakthroughs: first Shoen and Yau [SY1] using minimal surfaces, were able to solve the problem in low dimensions and then independently Gromov and Lawson [GL1,2,3], using twisted harmonic spinors resolved it for all dimensions. In the next section we will discuss some newer results on scalar curvature rigidity obtained by spinorial methods.

In the positive mass theorem, if we ignore the fact that the space-like slice $\left(M^{3}, g\right)$ is imbedded in space-time, the assumption to be asymptotically Euclidean is well defined by (1) and the formula (3) for the energy (or mass) $E$ still makes sense. In this Riemannian situation, the appropriate assumption that replaces the dominant energy condition (B) is that the scalar curvature of $\left(M^{3}, g\right)$ is non-negative. In fact, if the space-like slice has zero mean curvature zero $(\operatorname{tr}(h)=0)$, then by the Gauss-Codazzi equations, assumption (B) would imply that the scalar curvature of $M^{3}$ is non-negative. More generally, one would like to pose the problem whether, for all dimensions $n$, an asymptotically Euclidean Riemannian manifold $\left(M^{n}, g\right)$ with non-negative scalar curvature has non-negative mass . This is also pertinent to physics, if one works in a more general framework than classical relativity. To be exact, one needs to modify the decay rate in definition (1), depending on $n$. We assume for simplicity:

$$
\partial_{\alpha}\left(g_{i j}-\delta_{i j}\right) \in O\left(r^{-n+2-|\alpha|}\right) \text { for }|\alpha| \leq 2
$$

With this definition of asymptotically Euclidean we have the following result.

Theorem 2 An asymptotically Euclidean spin manifold $M$ with non-negative scalar curvature everywhere has positive total mass $E$. Moreover $E=0$ if and only if $M$ is isometric to flat Euclidean space.

Both Schoen-Yau and Witten established this theorem in dimension 3 in the course of their proof of the positive mass conjecture. For general $n$, this was first proved by Bartnik [B]. We note that for a compactly supported perturbation of the flat metric, the above theorem would be a simple consequence of the corresponding result for the torus.

It is a natural question to ask for similar results with different asymptotic geometries and in [M1], I established the following hyperbolic version of the rigidity part of theorem 2.

Theorem 3 A strongly asymptotically hyperbolic spin manifold of dimension $>2$, whose scalar curvature satisfies $R \geq-n(n-1)$ everywhere, is isometric to hyperbolic space.

The definition of strongly asymptotically hyperbolic in [M1] is rather rigid and would correspond, in the Euclidean version, to the zero mass case. In this respect, it should be 
interesting to understand the Bondi mass, defined in relativity in a general mathematical context.

After dealing with the Euclidean and hyperbolic versions of local scalar curvature rigidity, it is natural to pose the question for the sphere. The sharp local rigidity theorem for the spherical metric is the following theorem on the hemisphere, which I have recently proved $[\mathrm{M} 2]$.

Theorem 4 Let $M^{n}$ be a compact spin manifold with simply connected boundary $\partial M$ and let $g$ be a Riemannian metric on $M$ with the following properties:

(i) $\partial M$ is totally geodesic in $M$;

(ii) the metric induced on $\partial M$ by $g$ has constant sectional curvature $K \equiv 1$;

(iii) the scalar curvature of $g$ satisfies $R(g) \geq n(n-1)$ everywhere on $M$.

Then $(M, g)$ is isometric to the round hemisphere with the standard metric.

\section{Global Rigidity}

The first global theorem on scalar curvature rigidity is the following well known result that any metric with non-negative scalar curvature on the torus is flat.

Theorem 5 Let $g$ be a Riemannian metric on $T^{n}$ with scalar curvature $R(g) \geq 0$ everywhere. Then $g$ is flat.

This was proved first by Schoen-Yau [SY1] for low dimensions $(\leq 7)$ and then by GromovLawson [GL1,2,3] in all dimensions. The proof by Schoen and Yau is a somewhat simpler version of their argument to establish the positive mass theorem and uses the second variation formula for stable minimal surfaces. The proof by Gromov-Lawson on the other hand, uses spinors. and is closer in spirit to Witten's proof. However, since the result now is global, the argument is more elaborate and is based on the Index Theorem. This was not needed in any of the local rigidity results. In a recent paper, Gromov [G] has given an elegant re-interpretion of the basic idea of their proof, expressing it as an inequality relating the scalar curvature to a new $C^{0}$-invariant of a Riemannian metric called the K-area. The $\mathrm{K}$-area is, roughly speaking, the inverse of the norm of the smallest curvature obtainable on the manifold in the class of all topologically essential unitary bundles equipped with a connection. We refer to $[\mathrm{G}]$ for the precise definition. The main technique is then to obtain an inequality bounding the $\mathrm{K}$-area from below, in terms of the inverse of the infimum of the scalar curvature, provided the manifold carries a metric with positive scalar curvature. This can be achieved by analyzing a Weitzenbock formula for the square of a twisted Dirac operator and appealing to the index theorem. Since there are almost flat but essential bundles on a large covering of a torus, we now see that a torus cannot carry a metric of 
positive scalar curvature. Some extra geometric work is then needed to get the full scalar curvature rigidity result for the torus.

It is then a natural question to find Riemanian manifolds which are "extremal" with respect to this K-area inequality. The first sharp result of this nature was obtained by Llarull [L1][L2] who made a careful analysis of the proof by Gromov and Lawson in the case of the sphere to obtain the following theorem on the scalar curvature rigidity of spheres.

Theorem 6 Let $g$ be a Riemannian metric on $S^{n}$ satisfying $g \geq \bar{g}$ on all 2-vectors and with scalar curvature $R(g) \geq R(\bar{g}) \equiv n(n-1)$ everywhere, where $\bar{g}$ is the standard metric of constant sectional curvature $K \equiv 1$. Then $g \equiv \bar{g}$ everywhere.

This is the main result in Llarull's work, where one can also find various extensions and generalizations. In the next section, we will sketch a simplified proof of Llarull's theorem in even dimensions. An appropriate modification of the proof yields the following result for compact hermitean symmetric spaces.

Theorem 7 Let $M^{2 n}$ be a compact Hermitian-symmetric space with non-zero Todd genus. Let $\bar{g}$ be the symmetric metric of constant scalar curvature $R(\bar{g})$ and let $\omega$ be the Kähler form. If $g$ is any Riemannian metric on $M$ satisfying $|\omega|_{g}<|\omega|_{\bar{g}}$ then there is a point on $M$ where the scalar curvatures satisfy $R(g)<R(\bar{g})$.

The assumption on the metrics in Llarull's theorem can be stated more geometrically by saying that all surfaces in $M$ have larger area with respect to $g$ than the standard metric $\bar{g}$. For Hermitian-symmetric spaces, we relax the assumption on the metrics and compare them only on the Kähler form, i.e., only the areas of holomorphic curves need to be compared.

\section{The Proofs}

The first basic step in proving all the results above is to establish a version of the Lichnerowicz' formula for the square of an appropriate Dirac operator. For local rigidity theorems, we solve for harmonic spinors which have the correct behaviour at infinity and then integrate by parts. The boundary integrals, in the limit, are then identified with the "total mass". For global rigidity results, involving an inequality for the K-area in terms of lower bounds on the scalar curvature, we use appropriate bundles to twist the ordinary Dirac operator, make careful estimates of the curvature terms that appear in the (generalized) Lichnerowicz formula and then appeal to the index theorem.

The famous formula of Lichnerowicz for the ordinary Dirac operator $D$ is:

$$
D^{2}=\nabla^{*} \nabla+\frac{R}{4}
$$

where $\nabla$ is the Levi-Civita connection and $R$ is the ordinary scalar curvature. 
If we now integrate (9) on a manifold with boundary, we obtain:

$$
\int_{M}\left(|\nabla \psi|^{2}+\frac{R}{4}|\psi|^{2}\right)+\int_{M}|D \psi|^{2}=\int_{\partial M}\left\langle\nabla_{\nu} \psi+\nu D \psi, \psi\right\rangle
$$

where $\psi$ is a spinor and $\nu$ is the unit outer normal vector of the boundary.

For a harmonic spinor satisfying $D \psi=0$, the boundary integral on the right-hand-side will be non-negative, provided that $R \geq 0$ and vanish iff $\psi$ is globally parallel. To prove Theorem 2, we prove first the existence of a harmonic spinor which is asymptotically parallel in the sense that it approaches a parallel spinor (with respect to the background flat metric) sufficiently fast. The limiting value of the boundary integral is then shown to be the mass $(=E)$ as defined by (3) when the boundary spheres go off to infinity. Rigidity follows from the fact that if the mass vanishes, we get a trivialization of the manifold by parallel spinors , since we get one for each asymptotic value.

To prove Theorem 3, one needs a connection $\widetilde{\nabla}$ that is flat for the standard hyperbolic space. There is a natural one, which I called a hyperbolic Cartan connection in [M1], which comes from imbedding hyperbolic space in Minkowski space and restricting the flat vector space parallelism of the surrounding vector space. This can be done "virtually" for any Riemannian manifold on the stabilized tangent bundle $T M \oplus 1$, except that the connection would not be flat unless the manifold is hyperbolic. For spin manifolds, we also obtain induced connections on associated spinor bundles. The modified Dirac operator $\widetilde{D}$, is then defined using these Cartan connections. We refer to [M1] for details. This is highly reminiscent of Witten's proof of the positive energy theorem where he also used the Levicivita connection of the surrounding space-time restricted to the asymptotically Euclidean space-like slice to define a modified Dirac operator. The curvature terms that appear in the corresponding Lichnerowicz formula for the square of Witten's Dirac operator involve more than just the scalar curvature. However, miraculously, the dominant energy condition together with Einstein's equation is exactly what is needed to prove that the integrand is non-negative. The boundary integrals are, of course, then identified, in the limit, with the total energy-momentum vector.

In the case of the Dirac operator $\widetilde{D}$, defined by a hyperbolic Cartan connection the analogue of Lichnerowicz' formula is obtained simply by replacing the Riemannian connection and curvature terms by their hyperbolic analogues.

$$
\widetilde{D}^{2}=\widetilde{\nabla}^{*} \widetilde{\nabla}+\frac{\widetilde{R}}{4}
$$

where $\widetilde{R}=R+n(n-1)$ is the hyperbolic scalar curvature.

The proof of Theorem 3 now proceeds exactly as in the Euclidean case. First solve for a hyperbolically harmonic spinor with good asymptotics and then integrate by parts.

It is now not hard to guess that Theorem 4 is proved by using a Dirac operator coming from a spherical Cartan connection which is flat for the sphere. The corresponding 
Lichnerowicz-formula for the spherical Dirac operator is:

$$
\widetilde{D}^{2}-e_{0} \widetilde{D}=\widetilde{\nabla}^{*} \widetilde{\nabla}+\frac{\widetilde{R}}{4}=\left(e_{0} \widetilde{D}\right)^{2}+(n-1) e_{0} \widetilde{D}
$$

where $\widetilde{R}=R-n(n-1)$ is the spherical scalar curvature.

There are some technical subtleties, since this operator is no longer self-adjoint as it was in the hyperbolic case and so the Lichnerowicz formula is somewhat complicated, as one can see. Moreover, since one also has to deal with a finite boundary instead of infinity, "integration by parts" is more involved and we refer to [M2] for details. One solves an appropriate elliptic boundary value problem. However, the boundary conditions imposed are "local" and not of the Atiyah-Patodi-Singer type. The proof therefore does not use the Index Theorem. In fact, all proofs, that I know of, for local rigidity do not involve index arguments. In contrast, all the results that are stated about global scalar curvature rigidity on compact manifolds rely on the index of Dirac operators.

We now present a rather detailed proof of Llarull's theorem for even dimensional spheres. This is a somewhat simplified version of his proof and generalizes easily to prove Theorem 7. Again the first basic step is to derive and estimate an appropriately twisted version of the Lichnerowicz' formula. For the purpose of local calculations, we may always assume, that the manifold $\mathrm{M}$ is spin. Let $\mathbb{S}(g)=\mathbb{S}^{+}(g) \oplus \mathbb{S}^{-}(g)$ denote the bundle of spinors of an even dimensional spin manifold $(M, g)$, so we have two spinor bundles with respect to the two metrics $g$ and $\bar{g}$, where $\bar{g}$ is the standard metric. We consider the twisted Dirac operator $D$ on the bundle $\mathbb{S}(g) \otimes E$, where we choose the coefficient bundle to be $E=\mathbb{S}^{+}(\bar{g})$ (or $\mathbb{S}^{-}(\bar{g})$ ), the spinor bundle with respect to the spherical, or more generally, a symmetric background metric $\bar{g}$. Here we use the metric $g$ and its Levi-Civita connection to define the Dirac operator on the spinors in $\mathbb{S}(g)$, but for the twisting bundles $\mathbb{S}^{ \pm}(\bar{g})$, the Levi-Civita connection of the metric $\bar{g}$ is used.

We will regard the two spinor bundles $\mathbb{S}(g) \approx \mathbb{S}(\bar{g})$ as isomorphic complex vector bundles over $M$, with two different metrics but more importantly, admitting two different Clifford multiplications by vectors and exterior forms on $M$. To distinguish the two distinct Clifford multiplications, we will denote them by: $\sigma \mapsto \bar{\gamma}(v) \sigma$ for the metric $\bar{g}$ and $\sigma \mapsto \gamma(v) \sigma$ for the metric $g$, where $v$ is a tangent vector (or more generally for $v \in \Lambda^{*}(T M)$ ).

After diagonalizing the metric $g$ with respect to $\bar{g}$, so that we have two orthonormal bases: $\left\{\bar{e}_{i}\right\}$ for $\bar{g}$ and $\left\{e_{i}=\frac{1}{\lambda_{i}} \bar{e}_{i}\right\}$ for $g$, we can define $\gamma\left(e_{i}\right)=\bar{\gamma}\left(\bar{e}_{i}\right)$ and extend it canonically to the whole Clifford algebra with respect to $g$ to give us a new representation on the same Hermitian vector space $\mathbb{S}$. $\gamma$ then satisfies $\gamma(u) \gamma(v)+\gamma(v) \gamma(u)=-2 g(u, v)$ and $\gamma(u)$ is skew adjoint.

The twisted Dirac operator is then explicitly given by the formula:

$$
D\left(\sigma_{1} \otimes \sigma_{2}\right)=\sum_{k=1}^{2 n}\left\{\gamma\left(e_{k}\right) \nabla_{e_{k}} \sigma_{1} \otimes \sigma_{2}+\gamma\left(e_{k}\right) \sigma_{1} \otimes \bar{\nabla}_{e_{k}} \sigma_{2}\right\}
$$

where $\left\{e_{k}\right\}$ is an orthonormal base for the tangent vectors with respect to the metric $g$, 
$\nabla$ is the Levi-Civita connection of $g, \bar{\nabla}$ is the Levi-Civita connection of the metric $\bar{g}$ and $\sigma_{1} \otimes \sigma_{2} \in \Gamma\left(\mathbb{S} \otimes \mathbb{S}^{+}\right)$.

To simplify notation, we denote the product connection by $\nabla$, i.e.:

$$
\nabla_{v}\left(\sigma_{1} \otimes \sigma_{2}\right)=\nabla_{v} \sigma_{1} \otimes \sigma_{2}+\sigma_{1} \otimes \bar{\nabla}_{v} \sigma_{2} .
$$

The Lichnerowicz formula for $D^{2}$ is then computed to be: (compare [LM, Chap.II, formula (8.23), p.164])

$$
D^{2}\left(\sigma_{1} \otimes \sigma_{2}\right)=\nabla^{*} \nabla\left(\sigma_{1} \otimes \sigma_{2}\right)+\frac{R}{4} \sigma_{1} \otimes \sigma_{2}+\mathcal{R}\left(\sigma_{1} \otimes \sigma_{2}\right)
$$

for $\sigma_{1} \otimes \sigma_{2} \in \Gamma\left(\mathbb{S} \otimes \mathbb{S}^{+}\right)$, where $\nabla^{*}$ is the $L^{2}$-adjoint of $\nabla, R$ is the scalar curvature of the metric $g$, and the last term is explicitly given by:

$$
\mathcal{R}\left(\sigma_{1} \otimes \sigma_{2}\right)=\frac{1}{2} \sum_{j, k=1}^{2 n} \gamma\left(e_{j}\right) \gamma\left(e_{k}\right) \sigma_{1} \otimes \bar{R} m\left(e_{j} \wedge e_{k}\right) \sigma_{2}
$$

where $\left\{e_{i}\right\}$ is an orthonormal base with respect to the metric $g$ for the tangent space $T_{p} M$ at the point in question, $\bar{R} m$ is the curvature tensor of $\bar{g}$ and $\gamma$ is Clifford multiplication for $g$. Using the curvature operator $\bar{R} o p$ which acts on 2 -forms by:

$$
\bar{R} o p\left(e_{i} \wedge e_{j}\right)=\sum \bar{g}\left(\bar{R}\left(e_{i}, e_{j}\right) e_{l}, e_{k}\right) e_{k} \wedge e_{l}
$$

we can rewrite this expression as:

$$
\mathcal{R}\left(\sigma_{1} \otimes \sigma_{2}\right)=-\frac{1}{2} \sum_{a=1}^{n(2 n-1)} \gamma\left(e_{a}\right) \sigma_{1} \otimes \bar{\gamma}\left(\bar{R} o p\left(e_{a}\right)\right) \sigma_{2}
$$

where $\left\{e_{a}\right\}$ is now an orthonormal base with respect to $g$ for $\Lambda^{2}\left(T_{p} M\right)$ and $\bar{R} o p$ is the curvature operator of the symmetric metric. We note the right hand sides of (15) and (16) are independent of the orthonormal bases chosen and $\mathcal{R}$ is a well-defined self-adjoint algebraic operator on $\mathbb{S} \otimes \mathbb{S}$. (Our notation for the curvature tensor is such that $g\left(R\left(e_{i}, e_{j}\right) e_{j}, e_{i}\right)$ is the sectional curvature of the plane spanned by $e_{i}$ and $e_{j}$ ). The curvature operator of the spherical metric $\bar{g}$ is just the identity map on 2-vectors, so

$$
\mathcal{R}\left(\sigma_{1} \otimes \sigma_{2}\right)=-\frac{1}{2} \sum_{a=1}^{m} \gamma\left(e_{a}\right) \sigma_{1} \otimes \bar{\gamma}\left(e_{a}\right) \sigma_{2}
$$

where $m=n(2 n-1)$.

Let $g$ now satisfy the condition: $g \geq \bar{g}$ on all 2-forms. This simply means that $g(v, v) \geq$ $\bar{g}(v, v)$ for all $v \in \Lambda^{2}(T M)$. This implies that operator $\mathcal{R}$ dominates the corresponding operator $\overline{\mathcal{R}}$ defined by:

$$
\overline{\mathcal{R}}\left(\sigma_{1} \otimes \sigma_{2}\right)=-\frac{1}{2} \sum_{a=1}^{n(2 n-1)} \bar{\gamma}\left(\bar{e}_{a}\right) \sigma_{1} \otimes \bar{\gamma}\left(\bar{e}_{a}\right) \sigma_{2}
$$


where $\left\{\bar{e}_{a}\right\}, a=1, \ldots, m_{s}$ is now an orthonormal base with respect to $\bar{g}$ in the sense that $\mathcal{R}-\overline{\mathcal{R}}$ is positive semi-definite on $\mathbb{S} \otimes \mathbb{S}$ with respect to the metric $g \otimes \bar{g}$ (and hence also w.r.t. $\bar{g} \otimes \bar{g}$ ). This is easily seen by choosing $\left\{e_{a}\right\}$ to be an orthonormal base of eigenforms that diagonalizes the metric $g$ with respect to the background metric $\bar{g}$ on $\Lambda^{2}\left(T_{p} M\right)$, so that $e_{a}=\frac{1}{\lambda_{a}} \bar{e}_{a}$ with $\lambda_{a} \geq 1$ and with $\gamma\left(e_{a}\right)=\bar{\gamma}\left(\bar{e}_{a}\right) . \overline{\mathcal{R}}$ is then given by:

$$
\overline{\mathcal{R}}\left(\sigma_{1} \otimes \sigma_{2}\right)=-\frac{1}{2} \sum_{a=1}^{n(2 n-1)} \frac{1}{\lambda_{a}}\left(\gamma\left(e_{a}\right) \sigma_{1} \otimes \bar{\gamma}\left(\bar{e}_{a}\right) \sigma_{2}\right)
$$

We now estimate the minimum eigenvalue of the operator $\overline{\mathcal{R}}$. The Casimir operator $\mathcal{C}$ of the representation on $\mathbb{S} \otimes \mathbb{S}$ induced by the isotropy representation is given by:

$$
\begin{aligned}
\mathcal{C}\left(\sigma_{1} \otimes \sigma_{2}\right) & =-\sum_{a=1}^{m}\left(\bar{\gamma}\left(\bar{e}_{a}\right)\right)^{2}\left(\sigma_{1} \otimes \sigma_{2}\right) \\
& =-\sum_{a=1}^{m}\left\{\left(\bar{\gamma}\left(\bar{e}_{a}\right)\right)^{2} \sigma_{1} \otimes \sigma_{2}+2 \bar{\gamma}\left(\bar{e}_{a}\right) \sigma_{1} \otimes \bar{\gamma}_{(}\left(\bar{e}_{a}\right) \sigma_{2}+\sigma_{1} \otimes\left(\bar{\gamma}\left(\bar{e}_{a}\right)\right)^{2} \sigma_{2}\right\} \\
& =2 m\left(\sigma_{1} \otimes \sigma_{2}\right)+4 \overline{\mathcal{R}}\left(\sigma_{1} \otimes \sigma_{2}\right)
\end{aligned}
$$

by the Clifford identity $\left(\bar{\gamma}\left(\bar{e}_{a}\right)\right)^{2}=-I d$.

Now $\mathcal{C}$ is positive semi-definite on $\mathbb{S} \otimes \mathbb{S} \approx \Lambda^{*}(T M)$. In fact, it is positive definite on all non-trivial irreducible components of the representation and is equal to zero only on the trivial representations that occur, in particular for $\Lambda^{0}$ and $\Lambda^{n}$. In any case, we therefore have the following basic algebraic estimate:

$$
\mathcal{R} \geq \overline{\mathcal{R}} \geq-\frac{m}{2} I d=-\frac{\bar{R}}{4} I d \geq-\frac{R}{4} I d
$$

provided $R=R(g) \geq 2 n(2 n-1)=R(\bar{g})=\bar{R}$. Moreover, $\mathcal{R}$ is strictly $>-\frac{R}{4} I d$ unless all the inequalities above are strict equalities at all points of $M$.

This yields, by the Lichnerowicz formula (14), a vanishing theorem for both $\mathbb{S}^{+}(\bar{g})$ and $\mathbb{S}^{-}(\bar{g})$ - valued harmonic spinors on the compact manifold M, provided that $g \geq \bar{g}$ on $\Lambda^{2}\left(T_{p} M\right)$ for each $p$ and $R(g) \geq R(\bar{g})$ everywhere, with strict inequality holding at least at one point.

By the Index Theorem the topological indices of the two twisted Dirac operators are given by:

$$
\int \hat{A}(M) \cdot \operatorname{ch}\left(\mathbb{S}^{ \pm}\right)=\frac{1}{2}(e(M) \pm \tau(M))
$$

where $e(M)$ is the Euler characteristic and $\tau(M)$ is the signature of $M$. This index is nonzero for even-dimensional spheres. Rigidity follows from the fact that if strict equality holds 
in the inequalities above we must have $g \equiv \bar{g}$ on $\Lambda^{2}{ }_{p}$ at all points. Since $g \geq \bar{g}$ on $\Lambda^{2}$ this now implies that the two metrics are identical on each tangent space. The odd dimensional case can be proved by applying the even-dimensional case to $M \times S^{1}$, which is given an appropriate metric with a long $S^{1}$. We refer to [L].

To prove Theorem 7 , we consider the twisted Dirac operator $D$ on the bundle $\mathbb{S}(g) \otimes E$, where we choose the coefficient bundle $E$ to be the line bundle $L(\bar{g})$, whose square is the canonical bundle of the Hermitean symmetric space. Here we use the metric $g$ and its LeviCivita connection to define the Dirac operator on the spinors in $\mathbb{S}(g)$, but for the twisting bundle $L(\bar{g})$, the connection induced by the Levi-Civita connection of the symmetric metric $\bar{g}$ is used. Theorem 2 is proved in exactly the same fashion. The expression (15) for the operator $\mathcal{R}$ acting on $\mathbb{S}(g) \otimes L$ simplifies to

$$
\mathcal{R}(\sigma \otimes l)=-\frac{\bar{R}}{4} \gamma(\hat{\omega}) \sigma \otimes \bar{\gamma}(\hat{\omega}) l
$$

since the curvature form of the line bundle $L$ is $\frac{\bar{R}}{2} \hat{\omega}$, where $\hat{\omega}=\frac{\omega}{|\omega|_{\bar{g}}}$.

Under the assumption that $|\omega|_{\bar{g}}>|\omega|_{g}$ the operator $\mathcal{R}$ dominates the corresponding operator $\overline{\mathcal{R}}$ defined by:

$$
\overline{\mathcal{R}}(\sigma \otimes l)=-\frac{\bar{R}}{4} \bar{\gamma}(\hat{\omega}) \sigma \otimes \bar{\gamma}(\hat{\omega}) l
$$

which in turn dominates $-\frac{\bar{R}}{4} I d$ so that $\mathcal{R}+\frac{\bar{R}}{4} I d$ is positive definite on $\mathbb{S} \otimes L$. The assumption $R \geq \bar{R}$ would then imply that there are no harmonic spinors b Lichnerowicz' formula. The tensor product of the spinor bundle with the line bundle, whose square is the inverseof the canonical bundle exists globally on any Hermitian manifold, and is the spinor bundle of the canonically associated Spin $_{c}$ structure. The index for the corresponding Dirac operator is given by the Todd genus which is non-zero for compact Hermitian symmetric spaces and Theorem 7 follows.

QED

\section{References}

[B ] R. Bartnik, "The mass of an asymptotically flat manifold", Comm. Pure Appl. Math. 39 (1986), 661-693.

[G ] M. Gromov: "Positive curvature, macroscopic dimension, spectral gaps and higher signatures", Functional Analysis on the eve of the 21st century, vol.II, Progress in Math., vol. 132, Birkhauser, Boston, (1996).

[GL1 ] M. Gromov and H.B. Lawson: "Spin and scalar curvature in the presence of a fundamental group I"; Ann. Math. 111, 209-230 (1980). 
[GL2 ] M. Gromov and H.B. Lawson: "The clasification of simply-connected manifolds of positive scalar curvature"; Ann. Math. 111, 423-486 (1980).

[GL3 ] M. Gromov and H.B. Lawson, "Positive scalar curvature and the Dirac operator on complete Riemannian manifolds", Publ. Math. I.H.E.S. 58 (1983), 295-408.

[LM ] H.B. Lawson, and M.-L. Michelsohn: "Spin Geometry"; Princeton Math. Series 38, Princeton, 1989.

[L1 ] M. Llarull: "Sharp estimates and the Dirac Operator"; M. Llarull, Sharp estimates and the Dirac operator, Math. Ann. 310 (1998), 55-71.

[L2 ]M. Llarull, Scalar curvature estimates for $(n+4 k)$-dimensional manifolds, Differential Geom. Appl. 6 (1996), no. 4, 321-326.

[M1 ] M. Min-Oo, "Scalar curvature rigidity of asymptotically hyperbolic spin manifolds", Math. Ann. 285 (1989), 527-539.

[M2 ] M. Min-Oo, "Scalar curvature rigidity of the hemisphere", Preprint, McMaster, 1996.

[M3 ] M. Min-Oo, "Scalar curvature rigidity of compact Hermitian symmetric spaces", Preprint, McMaster, 1996.

[PT ] T. Parker and C.Taubes, "On Witten's proof of the positive energy theorem", Comm. Math. Phys. 84 (1982), 223-238.

[SY1 ] R. Schoen and S.T. Yau, "Existence of minimal surfaces and the topology of 3dimensional manifolds with non-negative scalar curvature", Ann. Math. 110 (1979), 127-142.

[SY2 ] R. Schoen and S.T. Yau, "On the proof of the positive mass conjecture in general relativity", Comm. Math. Phys. 65 (1979), 45-76.

[SY3 ] R. Schoen and S.T. Yau, "The energy and linear-momentum of space-times in general relativity", Comm. Math. Phys. 79 (1981), 47-51.

[SY4 ] R. Schoen and S.T. Yau, "Proof of the positive mass theorem II", Comm. Math. Phys. 79 (1981), 231-260.

[W ] E. Witten, "A new proof of the positive energy theorem", Comm. Math. Phys. 80 (1981), 381-402.

Maung Min-Oo

Dept. of Mathematics, McMaster University

Hamilton, Ont., Canada L8S 4K1

e-mail: minoo@mcmail.mcmaster.ca 